\title{
SMART LIPOSOMAL CHITOSAN-BASED AUTOGEL WITH OFLOXACIN; A NEW CONTROLLED RELEASE DEVICE USED FOR TREATMENT OF CHRONIC PERIODONTITIS. A RANDOMIZED, DOUBLE-BLIND CONTROLLED CLINICAL TRIAL
}

\author{
Abeer S. Gawish* and May M. Bilal**
}

\begin{abstract}
Background: Chronic periodontitis is an inflammatory disease caused by groups of specific microorganisms. Localized problem sites raise the concept of use of local drug delivery as effective therapeutic modalities for the treatment of periodontal diseases. The aim of the present study was to evaluate the clinical and microbiological effect of a single administration of a smart controlledrelease liposomal autogel system of ofloxacin in adjunct to non- surgical therapy in the management of chronic periodontitis patients.
\end{abstract}

Materials and Methods: In a split mouth design, twenty patients suffering from chronic periodontitis and displaying at least two contra-lateral intrabony defects were randomly selected. Non-surgical treatment (subgingival scaling and root planing) was performed in all sites. One of the two forms of ofloxacin, liposomal autogel,was applied in twenty of the pockets. The other twenty pockets received non surgical periodontal therapy with ofloxacin solution and act as the control sites. The autogel based on chitosan neutralized by $\beta$ - glycerophosphate was characterized for mucoadhesion, syringeability and gelation onset. The gel, liposomes afforded $80 \%$ of drug release in 7 days. Clinical parameters; including plaque index, gingival index, bleeding on probing, probing depth, clinical attachment level (PI, GI, BOP, PD and CAL); were recorded at the base line and 3 months following the non- surgical periodontal therapy. In addition, microbiological examination at the baseline, 1,3 and 7 days after were done to assess the sustained release effect.

Results: The microbiological assessment revealed that ofloxacin liposomal autogel demonstrated markedly lower anaerobes bioburdenin subgingival samples than ofloxacin solution after 7 days. Moreover, the liposomal autogel formula showed significant improvement in the different clinical parameters evaluated after three months.

Conclusion: Based on the microbiological and clinical results of the present investigation, the developed ofloxacin liposomal autogel is thought to be promising in the management of chronic periodontitis.

KEYWORDS: ofloxacin; smart autogel; chitosan/ $\beta$-glycerophosphate, liposomes; controlled release; chronic periodontitis

* Prof. of Oral Medicine, Peridontology, Vice Dean in Faculty of Dentistry, Sinai University, Staff member in Faculty of Dental Medicine Girls' Azhar university, Cairo, Egypt

** Lecturer of Oral Medicine and Periodontology, Faculty of Dentistry in Sinai University, Staff Member in Faculty of Dentistry, Mansoura University 


\section{INTRODUCTION}

Chronic periodontitis is a multifactorial infectious disease of the periodontium occurring because of the presence of virulent periodontopathic bacteria in addition to an immune response ${ }^{(1)}$. Approximately 500 bacteria taxa inhabit periodontal pockets, which provide a moist, warm, nutritious and anaerobic environment for microbial colonization and multiplication ${ }^{(2)}$. Considerable research into the identification of organisms responsible for periodontitis has implicated Porphyromonas gingivalis, Prevotella intermedia, Eikenella corrodens, Fusobacrerium nucleatum, and Tanerella forsythus for chronic periodontitis ${ }^{(3)}$, however, the exact mechanisms of tissue destruction are not completely elucidated. These suspected periodontal pathogens have been shown to produce a large number of biological molecules that may act directly on host tissue and destroy its integrity. On the other hand, there is evidence suggesting that the multitude of inflammatory and immune mediators produced by the host may cause tissue injury ${ }^{(4)}$. Therefore, periodontal therapy is aimed to remove the bacterial deposits from the tooth surface and to shift the pathogenic microbiota to one compatible with periodontal health ${ }^{(5)}$.

Antimicrobial agents, used as adjunctive to different therapeutic approaches which include mechanical or surgical methods, are one of most appropriate ways. The main goal of antibiotic therapy is to establish a concentration of drug that inhibits these pathogenic bacteria ${ }^{(6)}$. With advances in understanding of the etiology and pathogenesis of periodontal disease, attention has been focused on local drug delivery systems. These include both sustained and controlled release polymeric systems which when inserted into the periodontal pocket, release antimicrobial agents above the minimum inhibitory concentration (MIC) for a sustained period of time. Thus, intra-pocket devices have high benefit to low risk ratio ${ }^{(7)}$. Moreover, non- surgical approach (which include mechanical scaling and root planing) with adjunct local antimicrobial therapy proved useful treatment of periodontitis ${ }^{(8)}$.
Ofloxacin is a fluroquinolone antibiotic that has shown marked antibacterial activity against periodontopathic bacteria ${ }^{(9)}$. Nevertheless, its use in treatment of periodontitis is not adequately reported in literature. The only available forms of ofloxacin reported for treatment of periodontitis was a controlled release strip coded PT-01 and made of poly (methacrylic acid) and hydroxypropylcellulose containing $10 \%$ ofloxacin that afforded $40 \%$ ofloxacin release in $1 \mathrm{~h}$ and $70 \%$ release in $8 \mathrm{~h}$ ${ }^{(10)}$. Additionally, Yamagami et al 1992 developed a newly water soluble controlled release insert containing ofloxacin as antibacterial agent Pt01 that resulted in resolution of the periodontal inflammation ${ }^{(11)}$. More recently, Kranti et al 2016 investigated the effectiveness of a single subgingival administration of bio-absorbable controlled release $0.1 \%$ ofloxacin gel as an adjunct to scaling and root planing in the treatment of chronic periodontitis ${ }^{(12)}$.

Moreover, Srirangarajan et al 2011 suggested that this type of delivery system could significantly influence the outcome of therapy ${ }^{(13)}$. Ofloxacin in carrier polymer ethyl cellulose showed extended spectrum of antimicrobial activity and sustained in vitro release for a period of 11 days and could be maintained above MIC for the entire period of release ${ }^{(6)}$. With this perspective, controlled release delivery systems that can elute the antimicrobial agent at therapeutic level within the periodontal pocket over extended periods are valued by most clinicians. Additionally, systems in the form of injectable gels are more privileged than solid devices in being biocompatible, biodegradable, mucoadhesive and hence no need for surgical removal after complete drug elution ${ }^{(4)}$. Moreover, thermoresponsive gelling systems are highly appreciated due to their ease of syringeability and high ability to fill the pocket ${ }^{(14)}$.

Over the past few decades, novel approaches for drug delivery have been developed to alter the pharmacokinetic and pharmacodynamics properties of active pharmaceutical ingredient and to fabricate new polymeric materials that respond to external stimuli, such as temperature, light and $\mathrm{pH}^{(15)}$. This 
environmental responsiveness can be used to favor the encapsulation/release of active molecules ${ }^{(16)}$. One of the most used nanosystems to encapsulate drug molecules are liposomes, which are spherical bilayer vesicles formed by dispersion of certain polar lipids in aqueous solvents ${ }^{(17)}$. They have the ability to act as targeted release-on-demand carrier systems for both water- and oil-soluble functional compounds such as antimicrobials, antioxidants, and bioactive ingredients ${ }^{(18)}$. Encapsulation of functional components in liposomes has been shown to increase their stability and maintain their activity in environments that typically lead to rapid degradation ${ }^{(15)}$. Furthering, they have high loading capacities for water-soluble components and are biocompatible, biodegradable, and nontoxic ${ }^{(19)}$. Liposomes generally carry a negative surface charge due to the prevalence of phosphatidylcholine (PC) as a raw material, hence, manufacturing of positively or neutrally charged liposomes requires use of positively charged polar lipids such as phosphatidyl ethanolamine (PE) ${ }^{(20,21)}$.

Chitosan, that exhibits high antimicrobial activity, is an indigestible polysaccharide and considered as polycationic due to the presence of amino groups. Because of its positive charge, it has been used previously to build secondary layers around dispersed particles such as emulsion droplets and biopolymeric particles ${ }^{(22,20,21,23)}$. Chitosan is obtained by alkaline deacetylation from chitin (poly-N-acetyl-D-glucosamine), a major structural polysaccharide in the exoskeleton of arthropods and the cell wall of fungi. Onsoyen1992 and then Chen et al 2001 reported formation of a variety of structures upon addition of chitosan to liposomes ${ }^{(24,22)}$. They speculated on the formation of so-called chitosomes, that is, chitosan-coated liposomes.

Chitosan/ $\beta$-glycerophosphate (C/ $\beta$-GP) system was recently explored as thermo-responsive gelling systems in many applications ${ }^{(25-29)}$ with benefits in being biocompatible, biodegradable, mucoadhesive, formulated at low polymer concentration, having reasonable gelation temperature and can sustain drug for a longer period of time. Chitosan is an excellent candidate vehicle in periodontics for its reported safety as a promising scaffold for tissue engineering $^{(30,31)}$ and its antimicrobial activity against periodontopathic bacteria ${ }^{(32)}$. The polyol base, $\beta$-GP, serves dual function: first, increase the $\mathrm{pH}$ of chitosan to the physiological range (6.27.3). Second, prevent immediate precipitation or gelation, but instead, allow for controlled hydrogel formation when an increase in temperature is imposed i.e. confer thermoresponsive character to chitosan ${ }^{(33,34)}$. It was also reported that periodontal films of ofloxacin containing chitosan showed an initial burst release of the drug by more than $40 \%$, whereas, the maximum drug release (96.38) over a period of ten days ${ }^{(35)}$.

Therefore, autogels with reasonable $\mathrm{pH}$ and temperature of gelation could be prepared after setting a high degree of deacetylation, i.e. $>90 \%$ for chitosan. Prior encapsulation of the antibiotic in vesicular or particulate systems should afford more controlled ofloxacin release whereby the concentration of the antibiotic could be maintained for a sufficient time (at least a week) above the MIC. The prolonged residence time of ofloxacin in the periodontal pocket, would be beneficial in decreasing the systemic side effects and the frequency of administration. Thus, the main objective of the present study was to evaluate the clinical and microbiological effect of the use of new designed smart intrapocket delivery system for ofloxacin with site-specific gelation and controlled release inpatients with chronic periodontitis.

\section{MATERIALS AND METHODS}

\section{Study population}

This parallel-design-double-blind study was conducted on twenty patients having moderate to severe chronic periodontitis. The participants were selected from the Outpatient clinic, referred to Oral Medicine and Periodontology Department, Faculty of Dentistry, Sinai University during June 2015- January 2016. 
Each patient was informed of the objectives and nature of the study, including benefits and risks, and was required to sign an informed consents prior participation in the study. Patient population consisted of twenty patients (12 female and 8 males) with an age ranged from 39-55 suffering from chronic periodontitis and exhibiting forty intrabony defects. The selected patients were systemically healthy, with no history of periodontal therapy in the past 6 months, and not receiving any medications or antibiotics known to affect the periodontal status during the past 6 months. Each patient had chronic periodontitis with at least two contralateral periodontal defects with clinical attachment loss $\geq 3 \mathrm{~mm}$.

Exclusion criteria included history of hypersensitivity or allergy to quinolones group; pregnancy or lactation; smokers; a history of alcohol abuse; and participation in other clinical trials.

\section{Studydesign}

\section{Initial visit}

Subjects who met the inclusion/exclusion criteria were assigned numbers in ascending order by the study coordinator and completed a written medical history, which was verbally. Vital signs were taken, together with comprehensive intraoral examination, by a single investigator.

\section{Clinical parameters}

The following clinical parameters which include; Plaque index (PI) -Silness \& LÖe $1964^{(36)}$, Gingival index -LÖe \& Silness $1963^{(37)}$, bleeding on probing (BOP) -Newbrun $1996^{(38)}$, probing depth (PD), and clinical attachment level (CAL) ${ }^{(39)}$; were recorded using graduated William's probe at base line and three months following the non- surgical periodontal therapy.

\section{Randomization procedure and treatment protocol}

All sites (forty intrabony pockets) were randomly assigned by the study coordinator, using a coin toss, to receive one of the two treatments. They were treated with single course of non-surgical instrumentation followed by administration of either the test formula i.e. ofloxacin liposomal autogel (20 pockets) or ofloxacin $(0.1 \%)$ solution in phosphate buffer $\mathrm{pH} 6.8$ to serve as positive controls. These formulations were prepared in the Department of Pharmaceutics, Faculty of Pharmacy, Ain Shams University. Both medications were coded by the pharmacist and given to the study coordinator who was the only person who had access to them. The randomization process led to comparable mean values of all investigated clinical parameters at base line in both groups.

All participants were given detailed instructions in self performed plaque control measures and instructed not to use any type of chemical plaque control.

\section{Microbiological evaluation}

Baseline sampling (subgingival plaque collection): After complete isolation and full mouth scaling (removing the supragingival plaque) and root planing, subgingival plaque samples were collected by inserting a sterile paper point into a periodontal pocket and kept for 30s. Each paper point was transferred within 1 or $2 \mathrm{~s}$ to $5 \mathrm{ml}$ thioglycolate transport medium in well closed screw capped bottles. The bottles were collected in anaerobic jar. ${ }^{(9,40,41,42)}$.

At the same session, experimental sites received a single subgingival application of ofloxacin liposomal autogel or ofloxacin solutions. The preparations were slowly delivered to the bottom of the periodontal pocket by using a disposable syringe equipped with a blunt needle until overflowed from the gingival margin. All food and drink were prohibited for $2 \mathrm{~h}$ after injection. Also, patients were instructed not to use mouthwashes, antibiotics or any anti-plaque agents during the observation interval. All pockets were sampled again at days 1,3 and 7 for culture studies to measure the bactericidal efficacy of ofloxacin liposomal autogels in comparison to ofloxacin solutions. 


\section{Determination of bacterial count}

The bacterial deposits were displaced from each paper point and dispersed by vortex mixing for 30s. The bacterial viable count was done using the agar diffusion technique employing the spread plate method ${ }^{(43)}$. One $\mathrm{ml}$ of the homogenized bacterial suspension was placed on sterile Colombia agar plates. The plates were incubated at $37^{\circ} \mathrm{C}$ for $48 \mathrm{~h}$ in an anaerobic jar where full anaerobiasis was achieved by using gas pack for the generation of hydrogen and carbon dioxide in the jar. The total colony forming units per $\mathrm{ml}(\mathrm{CFU} / \mathrm{ml})$ were counted on the Colombia agar plates $(9,40,41$, 42). Percentage reduction of bacterial count was calculated according to the following equation:

$\%$ reduction in bacterial count from baseline $=\frac{\mathrm{C} 0-\mathrm{Ct}}{\mathrm{C} 0} \times 100$

$\mathrm{C} 0=$ Bacterial count at day 0 (baseline line)

$\mathrm{Ct}=$ Bacterial count at day $\mathrm{t}(1,3$ and 7$)$

\section{Statistical Analysis}

Data are presented as means and standard deviation. One Way Analysis of Variance (Anova) is used to test the difference between the control and test groups, followed by the unpaired " $t$ '" test to determine whether there was a statistical significant change from baseline within each group and between the groups in the different parameters measured. The level of significant was set at $p<0.05$ for all statistical tests.

\section{RESULTS}

Twenty subjects participated in and completed this study without dropouts. All patients showed good compliance without any signs of inflammation or swelling, indicating the biocompatibility of the materials.

The mean values and standard deviation of the clinical parameters at the baseline and three months after treatment in the control and test groups were shown in table (1). The mean values of the plaque index in the control and test groups were $2.25 \pm$
0.44 and $2.25 \pm 0.36$, respectively at the baseline. On the other hand, there was no statistical significant difference in this index between both groups at the end of the treatment as all pockets showed no plaque (score 0).

Furthering, the mean values of the gingival index in the control group was $2.42 \pm 0.66$ at the baseline and $1.37 \pm 0.42$ after treatment. Regarding the test group, the mean values of this index before and after treatment were $2.5 \pm 0.52$ and 0 , respectively. The mean GI reduction was $1.05 \pm 0.24$ in the control group and $2.5 \pm 0.52$ in the test group. However, the improvement of the gingival condition in the test group was significantly different from the control group (unpaired t test, $\mathrm{p}<0.05$ ).

BOP frequencies before treatment were $88.33 \%$ and $100 \%$ in the control and test groups, respectively, and were significantly reduced to $50 \%$ and $0 \%$ at the end of the treatment (unpaired t test, $\mathrm{p}<0.05$ ). However, the reduction in BOP frequency in the test group was significantly different from the reduction in BOP frequency in the control group (unpaired $t$ test, $\mathrm{p}<0.05)$.

In addition, the PD in the test group showed a mean value of $4.41 \pm 1.48$ at the baseline, and $3.54 \pm 1.37$ after treatment, whereas, in the control group, the mean values before and after treatment were $4.92 \pm 1.09$ and $2.54 \pm 1.01$, respectively. Considering the CAL, the mean values in the test group at the baseline and after treatment were $3.41 \pm$ 1.16 and $3.13 \pm 1.17$, respectively. On the other hand, the mean values in the control group before and after treatmentwere $5.38 \pm 1.53$ and $3.33 \pm$ 0.83 , respectively. Moreover, mean PD reduction was $0.87 \pm 0.11$ and $2.38 \pm 0.08 \mathrm{~mm}$ in the control and test groups, respectively. This reduction was in parallel with the mean CAL gain which was about $0.28 \pm 0.01$ and $2.05 \pm 0.70 \mathrm{~mm}$ in the control and test groups, respectively. There was a statistical significant difference in both the mean PD reduction and the mean CAL gain between both groups at the end of the treatment. 
TABLE (1) The clinical parameters for control and test pockets receiving ofloxacin solution and liposomal autogel respectively at day 0 and after 3 months.

\begin{tabular}{|c|c|c|c|c|c|}
\hline \multicolumn{3}{|c|}{ Control Group } & \multicolumn{3}{|c|}{ Test Group } \\
\hline & Day 0 & 3 months after & & Day 0 & 3 months after \\
\hline Mean PI ${ }^{1}$ & $2.25 \pm 0.44$ & $0 *$ & Mean PI ${ }^{1}$ & $2.25 \pm 0.36$ & $0^{*}$ \\
\hline \multicolumn{6}{|c|}{ Mean PI reduction ${ }^{2}$} \\
\hline \multicolumn{3}{|c|}{$2.25 \pm 0.44^{\mathrm{a}}$} & \multicolumn{3}{|c|}{$2.25 \pm 0.36^{\mathrm{b}}$} \\
\hline Mean GI ${ }^{1}$ & $2.42 \pm 0.66$ & $1.37 \pm 0.42 *$ & Mean GI ${ }^{1}$ & $2.5 \pm 0.52$ & $0 *$ \\
\hline \multicolumn{6}{|c|}{ Mean GI reduction ${ }^{2}$} \\
\hline \multicolumn{3}{|c|}{$1.05 \pm 0.24^{\mathrm{c}}$} & \multicolumn{3}{|c|}{$2.5 \pm 0.52^{\mathrm{d}}$} \\
\hline $\begin{array}{c}\text { (BOP) } \\
\text { frequency }\end{array}$ & $83.33 \%$ & $50.00 \% *$ & $\begin{array}{c}(\mathrm{BOP}) \\
\text { frequency }\end{array}$ & $100 \%$ & $0 \% *$ \\
\hline \multicolumn{6}{|c|}{ (BOP) frequency reduction ${ }^{2}$} \\
\hline \multicolumn{3}{|c|}{$33.33 \%^{\mathrm{c}}$} & \multicolumn{3}{|c|}{$100 \%^{\mathrm{d}}$} \\
\hline Mean PD & $4.41 \pm 1.48^{\mathrm{a}}$ & $3.54 \pm 1.37^{\mathrm{b}}$ & Mean PD & $4.92 \pm 1.09$ & $2.54 \pm 1.01 *$ \\
\hline \multicolumn{6}{|c|}{ Mean (PD) reduction ${ }^{2}$} \\
\hline \multicolumn{3}{|c|}{$0.87 \pm 0.11^{\mathrm{C}}$} & \multicolumn{3}{|c|}{$2.38 \pm 0.08^{\mathrm{d}}$} \\
\hline Mean CAL & $3.41 \pm 1.16^{\mathrm{a}}$ & $3.13 \pm 1.17^{\mathrm{b}}$ & Mean CAL & $5.38 \pm 1.53$ & $3.33 \pm 0.83 *$ \\
\hline \multicolumn{6}{|c|}{ Mean (CAL) gain ${ }^{2}$} \\
\hline \multicolumn{3}{|c|}{$0.28 \pm 0.01^{\mathrm{c}}$} & \multicolumn{3}{|c|}{$2.05 \pm 0.70^{\mathrm{d}}$} \\
\hline
\end{tabular}

${ }^{1}$ PI (Plaque Index) and GI (Gingival Index) = total score/number of surfaces examined.

${ }^{2}$ Mean reduction or gain $=$ difference between the mean at baseline and after 3 months of treatment. ${ }^{a, b}$ not significantly different (unpaired 1 test, $p=1.0000$ ). ${ }^{c, d}$ significantly different (unpaired t test, $P<0.05$ ). *significantly different from the corresponding base line (unpaired t test, $P<0.05$ ).

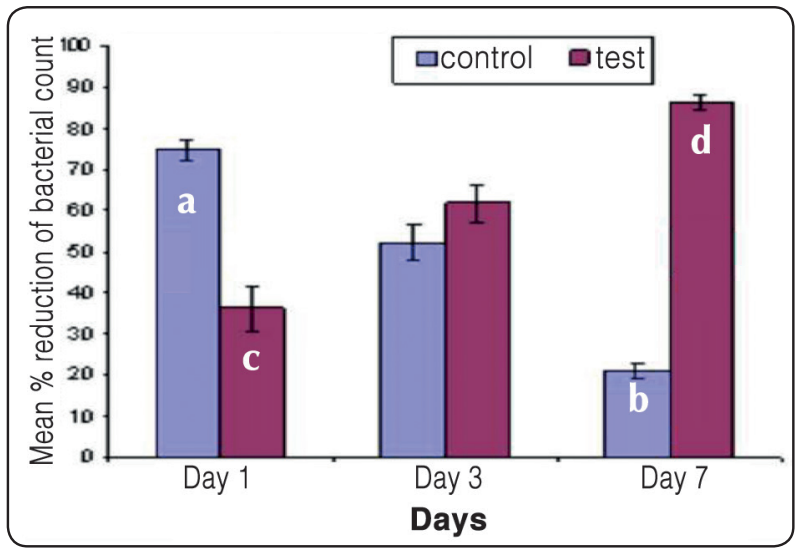

Fig. (1): The mean \% reduction in bacterial count in both groups. a and b show significant difference (unpaired $\mathrm{t}$ test, $\mathrm{p}<0.05)$. $\mathrm{c}$ and $\mathrm{d}$ show significant difference (unpaired $\mathrm{t}$ test, $\mathrm{p}<0.05$ ). a and $\mathrm{d}$ show significant difference (unpaired t test, $\mathrm{p}<0.05$ ).
Regarding the microbiological results, the bacterial count of subgingival plaque at each periodontal site was performed at day $0,1,3$ and 7 as shown in figure (1). Periodontal pockets in the test group (receiving ofloxacin liposomal autogel) had mean bacterial percentage reduction of $36.13 \%$, $61.78 \%$ and $86.20 \%$, while for those in the control group (receiving ofloxacin solution), the mean bacterial percentage reduction values were $74.74 \%$, $52.09 \%$ and $20.75 \%$ at day 1,3 and 7 respectively, confirming a long lasting antibacterial action in case of liposomal autogel. The antibacterial effect of ofloxacin liposomal autogel $(86.20 \%$ bacterial reduction; day 7) was significantly higher $(\mathrm{p}<0.05)$ than the antibacterial effect of ofloxacin solution (maximum bacterial reduction $74.2 \%$; day 1 ). 


\section{DISCUSSION}

Scaling and root planing in conjunction with proper plaque control results in alteration of the subgingival environment that is sufficient, in most instances to improve periodontal health and arrest further loss of attachment. Nevertheless, scaling and root planing alone may not predictably lead to complete elimination of the disease ${ }^{(44)}$. Poor access to the bottom of deep pockets and anatomical complexities may occasionally limit the efficacy of root planing. Moreover, some bacteria have been shown to invade deep periodontal tissues, making mechanical therapy alone sometimes ineffective ${ }^{(45)}$ and repopulation of scaled teeth from bacterial reservoirs in dentinal tubules may also be responsible for recurrence of the disease ${ }^{(46)}$.

Various antimicrobial agents have been administered systemically as well as locally/topically by means of mouth rinses or irrigation solutions as an adjunct to scaling and root planning ${ }^{(47)}$. However, systemic administration of antibiotics have been associated with side effects, while effectiveness of local delivery of antimicrobial agents in form of mouth rinses and subgingival irrigation has been limited due to inability of the drug to reach the site of action in adequate concentrations and the inability to localize and sustain at disease active sites ${ }^{(45)}$. Recently, advances in local delivery technology have resulted in control release of drugs that are successful in maintaining effective drug concentration at a lower dosage in the periodontal pocket.

In conjunction with mechanical removal of bacteria and bacterial toxins located on the roots, it appears that the use of smart controlled-release liposomal autogel system of ofloxacin led to reduction of clinical parameters and the bacterial count of subgingivalplaque in chronic periodontitis. Ofloxacin is considered as one of the synthetic pyridine carboxylic acid (PCA) derivatives. Although the earlier PCA derivatives were not active against Gram positive bacteria and anaerobes, ofloxacin can kill Gram positive bacteria and anaerobic bacteria and showed marked antibacterial activity against periodontopathic bacteria including Bacteroides species, Fusobacterium species and Actinobacillus actinomycetemcomitans ${ }^{(9)}$. Furthermore, the uptake of ofloxacin by resting polymorphonuclear leukocytes (PMNs) appears to be much higher than the uptake of other quinolones. PMNs may serve as vehicles for transport and delivery of fluoroquinolones as they migrate from the blood stream to infection sites. By this mechanism, PMNs have the potential to enhance resolution of an infection by increasing the local quinolone concentration at sites most beneficial to the host ${ }^{(48)}$. It was also reported that ofloxacin had high chemical stability and rare adverse effects ${ }^{(9)}$.

Regarding the clinical results, it is worth to report that all the patients receiving ofloxacin liposomal autogel reported no complaints or signs of allergy, inflammation, irritation, pus formation or any other complications suggesting that this formulation as well as ofloxacin solution were well tolerated. Similar results were reported by the study of Higashi et al 1990 who evaluated the effectiveness of controlled-release insert PT-01 and its release profile ${ }^{(10)}$. The autogel preparation was easy in clinical handling, manipulation and easily injectable inside the pockets. Furthering, it was fluid enough to allow subgingival placement using a simple syringe, requiring only a few seconds to completely fill the periodontal pocket. All pockets (test and control) showed no plaque three months after injection. This might be attributed to plaque removal which was done at the baseline and was followed by constant reinforcement of oral hygiene instruction at each visit. So, there was none significant difference (unpaired t test, $p=1.0000$ ) in the effect of both ofloxacin liposomal autogel and ofloxacin solution on the plaque index. However, the mean GI reduction and reduction in BOP frequency, for pockets in the test group were significantly higher (unpaired $t$ test, $\mathrm{P}<0.05$ ) than those in the control group. Thus, the sustainment of ofloxacin 
in the pocket for a week was more advantageous in reducing gingival inflammation than ofloxacin solution. Additionally, statistical analysis showed that treatment with ofloxacin liposomal autogel achieved extremely significant PD reduction and CAL gain in contrast to the treatment with ofloxacin solution (unpaired t test, $\mathrm{p}<0.05$ ). This could be simply explained by the fact that gel formulation can be easily administered and have relatively faster drug release at the site of application and was also bioadhesive and biocompatible with the oral mucosa (5). These results were consistent with the results of the study of Yamagamietal $1992^{(11)}$ who reported that weekly application of a water soluble controlled release insert containing ofloxacin PT-01 had a significant effect on the reduction of inflammation in patients with chronic periodontitis. Additionally, similar results were reported by Kranti et al 2016 after single subgingival administration of bioabsorbable controlled release $0.1 \%$ of ofloxacin gel as an adjunct to scaling and root planing resulting in additional PD reduction compared to scaling and root planing alone ${ }^{(12)}$.

Hence, based on the fact that our formula has achieved mean PD reduction of $2.38 \mathrm{~mm}$ and mean CAL gain of $2.05 \mathrm{~mm}$, it is clear that this formula achieved clinical improvements greater than those achieved by the marketed products as mentioned in the study of Arthur et al $2004{ }^{(49)}$.

Regarding the microbiological results, the use of ofloxacin liposomal autogel resulted in $86.20 \%$ bacterial reduction at day 7 , whereas, the maximum effect of ofloxacin solution on bacterial reduction was $74.2 \%$ at day 1 . Therefore, the antibacterial effect of gel formulation was significantly higher (unpaired $\mathrm{t}$ test, $\mathrm{P}<0.05$ ) than that of the solution. These results were consistent with those of Kimura et al $1991^{(9)}$ who reported a significant reduction of the total viable counts of bacteria, black-pigmented Bacteroides and Fusobacterium species with the controlled-release insert containing ofloxacin PT- 01 . In fact, the mean percentage reduction of $86.20 \%$ observed after one week treatment with ofloxacin liposomal autogel was considered significantly higher than the reported reduction in bacterial count in the previous studies ${ }^{(9,42)}$. Hence, it can be concluded that the sustained release achieved by liposomal $\mathrm{C} / \beta$-GP was important for the bactericidal efficacy of ofloxacin. In addition, the antibacterial effect of chitosan itself might have shared in this high bacterial reduction ${ }^{(50-53)}$. It was also proved the bactericidal effect of chitosan based thermosensitive hydrogel against the periodontopathic bacteria ${ }^{(32)}$. Hence, three factors might have shared in this extremely significant bacterial count reduction viz, the use of the powerful ofloxacin antibiotic, the use of the bactericidal, slowly eroding, $\mathrm{C} / \beta-\mathrm{GP}$ as a vehicle and the sustained release obtained by the liposomal inclusion into the thermoresponsiveautogel.

In the present study, ofloxacin liposomal autogel had proved a greater efficacy in treatment of periodontitis. Two factors might have shared in this significant clinical improvement viz, the significant reduction in bacterial count shown before and the cell proliferation and tissue regeneration-promoting ability of chitosan per se. This proved that $\mathrm{C} / \beta-\mathrm{GP}$ was an excellent candidate vehicle in treatment of periodontitis.

\section{CONCLUSION}

After mechanical debridement, this smart liposomal chitosan-based auto gel with ofloxacin system markedly suppressed the anaerobes bioburden in the subgingival milieu for at least a week and released therapeutic levels of ofloxacin in the periodontal pockets resulting in improvement in all clinical parameters three months post-treatment and proving itssuperiority over ofloxacin solution.

\section{RECOMMENDATION}

More investigation and long-term studies using this smart system with different concentrations for treatment of other forms of periodontal diseases are suggested to be carried out to affirm the results of our study. 


\section{REFERENCES}

1. Greenstein, G., 2006. Local delivery in the treatment of periodontal disease: Assessing the clinical significance of the results. J Periodontol. 77, 565-578.

2. Walker, C. B., Karpinia, K., Baehni, P., 2004. Chemotherapeutics: antibiotics and other antimicrobials. Periodontol 2000. 36, 146-165.

3- Haffajee, A.D., Socransky, S.S., 1994. Microbial etiological agents of destructive periodontal disease, Periodontol 2000.5, 78-111.

4- Schwach-Abdellaoui, K., Vivien-Castioni, N., Gurny, R., 2000. Local delivery of antimicrobial agents for the treatment of periodontal diseases. Eur J Pharm Biopharm. 50(1), 83-99.

5- Raheja, I., Kohli K., Drabu S., 2013. Periodontal drug delivery system containing antimicrobial agents. Int J Pharm. Sci. 5(3), 11-16.

6- Ali, S., Ali, J., Ahuja, A., Alam, S., 2012. Formulation and characterization of dental film containing ofloxacin. Journal of Applied Pharmaceutical Science. 2 (11): 114-119.

7- Pandit, J. K., 2004. Targeted Devices For Periodontal Disease. In N K Jain (Ed) Controlled and novel drug delivery (pp. 130). CBS Publishers and distributors, New Delhi, India.

8- Jorgensen, D. D. S., Slots, D. D. S., 2000. Efficient antimicrobial treatment in periodontal maintenance care. J Am Dent Assoc.131(9), 1293-1304.

9- Kimura, S., Toda, H., Shimabukuro, Y., Kitamura, M., Fujimoto, N., Miki, Y., Okada, H., 1991. Topical chemotherapy in humanperiodontitis using a new controlled release insert containing ofloxacin. I. Microbiological observation. J Periodontal Res. 26,33-41.

10- Higashi, K., Morisaki, K., Hayashi, S., Kitamura, M., Fujimoto, N.,Kimura, S., Ebisu, S., Okada, H., 1990. Local ofloxacin delivery using a controlled-release insert, PT-01 in the human periodontalpocket. J Periodontal Res. 25, 1-5.

11- Yamagami, H., Takamori, A., Sakamoto, T., Okada, H., 1992. Intrapocket chemotherapy in adult periodontitis using a new controlled-release insert containing ofloxacin (PT-01). J Peridontol. 63(1), 2-6.

12- Kranti, K., Mani, R., Ashutosh, R., Tervankar A. R., 2016. Effectiveness of subgingivally delivered $0.4 \%$ moxifloxacin gel and $0.1 \%$ oflaxacin gel in the treatment of chronic periodontitis. IJDOH. 2(1):53-58.
13- Srirangarajan, S., Raghavendra, C., Mundargi, S., Swati, B., Setty, T. M., Aminabhavi, F., Srinath, T., 2011. Randomized, controlled, single-masked, clinical study to compare and evaluate theefficacy of microspheres and gel in periodontal pocket therapy. J Periodontol. 82(1): 114-121.

14- Mulik, R., Kulkarni, V., Murthy R. S. R.,2009.Chitosanbased thermosensitive hydrogel containing liposomes for sustained delivery of cytarabine. Drug Development and Industrial Pharmacy. 35:49-56.

15- Nagpal, K., Singh, S. K., Mishra, D. N., 2010. Chitosan nanoparticles: A promising system in novel drug delivery. Chem Pharm Bull. 58(11): 1423-1430.

16- Joglekar, M., Trewyn, B. G., 2013. Polymer-based stimuli responsive nanosystems for biomedical applications. Biotechnol J. 8(8): 931-945.

17- Benech, R. O., Kheadr, E. E., Lacroix, C., Fliss, I., 2002. Inhibition of Listeria innocuain Cheddar cheese by addition of nisin $\mathrm{Z}$ in liposomes or by in situ production in mixed culture. Appl Environ Microbiol. 68:3683-3690.

18- Were, L. M, B. D. B., Davidson, P. M., Weiss, J., 2003. Size, stability, and entrapment efficiency of phospholipid nanocapsules containing polypeptide antimicrobials. J Agric Food Chem. 51:8073-8079.

19- Gomez-Hens, A., Fernandez-Romero, J. M., 2006. Analytical methods for the control of liposomal delivery systems. Trends Anal Chem. 25(2):167-178.

20- Ogawa, S., Decker, E. A., McClements, D. J., 2003. Influence of environmental conditions on the stability of oil-in-water emulsions containing droplets stabilized by lecithinchitosan membranes. J Agric Food Chem. 51(18): 5522-5527.

21- Aoki, T., Decker, E. A.,McClements, D.J., 2005. Influence of environmental stresses on stability of $\mathrm{O} / \mathrm{W}$ emulsions containing droplets stabilized by multilayered membranes produced by a layer-by-layer electrostatic deposition technique. Food Hydrocolloids. 19:209-220.

22- Chen, R. H., Win, H. P., Fang, H. J., 2001. Vesicle size, size distribution, stability, and rheological properties of liposomes coated with water-soluble chitosans of different molecular weights and concentrations. J Liposome Res. 11(2-3): 211-228.

23- Klinkesorn, U., Sophanodora, P., Chinachoti, P., Decker, E. A., McClements, D. J., 2005. Stability of spray-dried tunao oil emulsions encapsulated with two-layered interfacial membranes. J Agric Food Chem. 53(21):8365-8371. 
24- Onsoyen, E., 1992. Chitosan as stabilizer and bioadhesive carrier inliposome formulation, in: C.J. Brine, P.A. Sandford, J.P. Zikakis, Eds., Advances in chitin and chitosa, Elsevier, London.479-488.

25- Gordon, S., Teichmann, E., Young, K., Finnie, K., Rades, T., Hook, S.,2010. In vitro and in vivo investigation of thermosensitive chitosanhydrogels containing silica nanoparticles for vaccine delivery. European Journal of Pharmaceutical Sciences. 41(2): 360-368.

26- Ji, Q. X., Zhao, Q. S., Deng, J., Lu, R., 2010. A novel injectable chlorhexidine thermosensitive hydrogel for periodontal application:preparation, antibacterial activity and toxicity evaluation. J Mater Sci Mater Med. 21(8): 2435-2442.

27- Kim, S., Nishimoto, S. K., Bumgardner, J. D., Haggard, W. O., Gaber, M.W., Yang, Y., 2010. A chitosan/ $\beta$-glyce rophosphatethermosensitivegel for the delivery of ellagic acid for the treatment ofbrain cancer. Biomaterials. 31(14): 4157-4166.

28- Rossi, S., Marciello, M., Bonferoni, M. C., Ferrari, F., Sandri, G.,Dacarro, C., Grisoli, P., Caramella, C., 2010. Thermally sensitivegels based on chitosan derivatives for the treatment of oralmucositis. Eur J Pharm Biopharm. 74(2): 248-254.

29- Zheng, L., Ao, Q., Han, H., Zhang, X., Gong, Y., 2010. Evaluation of the chitosan/glycerol-beta-phosphate disodium salt hydrogel application in peripheral

30- Crompton, K. E., Goud, J. D., Bellamkonda, R. V., Gengenbach, T. R., Finkelstein, D. I., Horne, M. K., Forsythe, J. S., 2007. Polylysine-functionalised thermoresponsive chitosan hydrogel for neuraltissue engineering. Biomaterials. 28(3): 441-449.

31- Yan, J., Yang, L., Wang, G., Xiao, Y., Zhang, B., Qi, N., 2010. Biocompatibility evaluation of chitosan-based injectable hydrogelsfor the culturing mice mesenchymal stem cells in vitro. J Biomater, Appl. 24(7): 625-637.

32- Ji, Q. X., Deng, J., Yu, X. B., Xu, Q. C., Xu, X. Y., 2009. An in vitro evaluation of the antibacterial activity of chitosanbased thermosensitive hydrogel against periodontal pathogens. ShanghaiKou Qiang Yi Xue. 18(4): 397-400.

33- Chenite, A., Chaput, C., Wang, D., Combes, C., Buschmann, M. D.,Hoemann, C. D., Leroux, J. C., Atkinson, B. L., Binette, F.,Selmani, A., 2000. Novel injectable neutral solutions of chitosan form biodegradable gels in situ. Biomaterials. 21 (21): 2155-2161.
34- Chenite, A., Buschmann, M. D., Wang, D., Chaput, C., Kandani, N., 2001. Rheological characterization of thermogelling chitosan/ glycerol-phosphate solutions. Carbohydr. Polym.46: 39-47.

35- Singh, N., Malviya, R., Bansal, M., Sharma, P., K., 2010. Formulation and evaluation of different polymer based periodontal film of ofloxacin. Der Pharmacia Letter. 2(3):297-303.

36- Silness, P., LÖe, H., 1964. Periodontal disease in pregnancy. Acta Odontologica Scanadinavica.22, 121.

37- LÖe, H., Silness, P., 1963. Periodontal disease in pregnancy. Acta Odontologica Scanadinavica.21, 533.

38- Newbrun, E., 1996. Indices to measure gingival bleeding. J Periodontol. 67: 555-561.

39- Carranza, F. A., Takei, H. H., 2014. Chapter 29: clinical diagnosis. In: textbook of Carranza's clinical periodontology: $12^{\text {th }}$ edition, p.357. Saunders Company.

40- Esposito, P., Cortesi, R., Cervellati, F., Menegatti, E., Nastruzzi, C.,1997. Biodegradable microparticles for sustained delivery oftetracycline to the periodontal pocket: formulatory and drug release studies. J Microencapsul. 14: 175-187.

41- David, S., Jonesa, A., Woolfsona, D., Andrew, F., Browna, Wilson, A.,Coulterb, Cathy McClelland, Christopher, R., Irwin., 2000. Design, characterization and preliminary clinical evaluation of a novel mucoadhesive topical formulation containing tetracycline for the treatment of periodontal disease. J Cont Rel. 67: 357-368.

42- Raghavendra, C., Mundargi, A. S., Srirangarajan, Sunil, A. A., Sangamesh, A. P. S., Ravindra, S. S., 2007. Development andevaluation of novel biodegradable microspheres based on poly, D, L-lactide-co-glycolide and poly, $\varepsilon$-caprolactone for controlled delivery of doxycycline in the treatment of human periodontal pocket: In vitro and in vivo studies. J Cont Rel. 119:59-68.

43- Jacquelyn, G. B., 1996. Microbiology: principles and applications. Chapter 3, pp. 140-143.

44- Aimetti, M., Romano, F., Torta, I., Cirillo, D., Caposio, P., Romagnoli, R., 2004. Debridement and local application of tetracycline-loaded fibres in the management of persistent periodontitis: results after 12 months. J ClinPeriodontol. 31:166-172.

45- Saglie, R., Newman, M. G., Carranza, F. A., Jr Pattison G. L., 1982. Bacterialinvasion of gingiva in advanced periodontitis in humans. J Periodontol. 53:217-222. 
46- Radvar M., Pourtaghi N., Kinane D.,F., 1996. Comparison of 3 periodontallocal antibiotic therapies in persistent periodontal pockets.J Periodontol.67:860-865.

47- Jeffcoat M. K., Bray K. S., Ciancio S. G., Dentino A. R., Fine D. H., GordonJ. M., et al., 1998. Adjunctive use of a subgingival controlled releasechlorhexidine chip reduces probing depth and improves attachmentlevel compared with scaling and root planing alone. J Periodontol. 69:989-997.

48- Kleinfelder J. W., Mueller R. F., Lange D. E., 2000. Fluoroquinolones in the treatment of Actinobacillusactinomy cetemcomitansassociatedperiodontitis. J Periodontol. 71(2):202-208.

49- Arthur, R. V., Jonathan, G., Elyzabeth, H., 2004. The periodonticSyllabus. Chapter 10, pp. 107-111.
50- Chung, Y. C., Ya-ping, S. U., Chen, C. C., Guang, J., Wang, H. L., Gaston, J. C., LIN, J. G., 2004. Relationship between antibacterial activity of chitosan and surface characteristics of cell wall. Acta Pharmacol Sin. 25(7): 932-936.

51- MohyEldin, M. S., Soliman, E. A., Hashem, A. I., Tamer, T. M., 2008. Antibacterial activity of chitosan chemically modified with newtechnique. Trends Biomater Artif Organs. 22(3): 125-137.

52- Xing, K., Chen, X. G., Li, Y. Y., Liu, C. S., Liu, C. G., Cha, D. S., Park,H. J., 2008. Antibacterial activity of oleoylchitosan nanoparticles:A novel antibacterial dispersion system. Carbohydr.Polym. 74(1):114-120.

53- Vsconez, M. B., Flores, S. K., Campos, C. A., Alvarado, J., Gerschenson, L. N., 2009. Antimicrobial activity and physical properties ofchitosan-tapioca starch based edible films and coatings. Food ResInt. 42(7): 762-769. 